\title{
Study of forming and Welding performance of Y-shaped transition ring for large launch vehicle tank
}

\author{
Zhilong Chang ${ }^{1, \mathrm{a}}$, Zhenggen $\mathrm{Hu}^{1}$, Cheng Huang ${ }^{1}$, Li Zhou ${ }^{2}$, Guanri Liu ${ }^{1}$, and Yihua Mo ${ }^{1}$ \\ ${ }^{1}$ Beijing Aerospace System Engineering Research Institute, 100076 Beijing, China \\ ${ }^{2}$ Shandong Provincial Key Laboratory of Special Welding Technology, 264209 Weihai, China
}

\begin{abstract}
As the critical load-bearing part of launch vehicle tank, the Y-shaped transition ring is manufactured by integral forging process currently, and it puts forward very high requirements for the forming precision, microstructure and welding performance of the integral forged transition ring. In this paper, the integral forging process for the Y-shaped transition ring of large launch vehicle tank is studied. Microstructure, mechanical properties and welding performance of the transition ring are analyzed. Improvement measures for integral forging process of large Y-shaped transition ring are proposed.
\end{abstract}

\section{Introduction}

Launch vehicle technology decides a country's ability to enter into space and development potential in the future, which is the indispensable guaranty for significant improvement of overall level of national space science and technology. The development of large launch vehicle technology is the symbol of a nation's comprehensive strength. With increasing launch vehicle size, the dimensional effects will be more obvious and the effects of manufacturing process on the structure performance will be more complex. As for the development of large launch vehicle, the design and manufacture for tank which occupies over $60 \%$ of the weight of the whole launch vehicle is the key, whilst the forming and welding of Y-shaped transition ring is critical for tank manufacture. The Y-shaped transition ring connects the dome, tube and short shell of tank as illustrated in Fig. 1, which bears complex stress and is the most important part of tank.

The manufacturing technology of Y-shaped transition ring for tank has been developing with the increasing requirements for launch vehicle performance, which could be generally divided into three stages in domestic and aboard:

1. profile roll forming + welding, which was gradually abandoned by aerospace powers such as Europe, US, Japan due to its disadvantages;

2. integral forging forming, which is improved based on profile roll forming + welding way and is commonly adopted currently though some defects still exist;

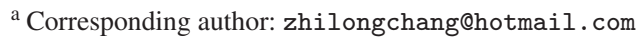

This is an Open Access article distributed under the terms of the Creative Commons Attribution License 4.0, which permits unrestricted use, distribution, and reproduction in any medium, provided the original work is properly cited. 


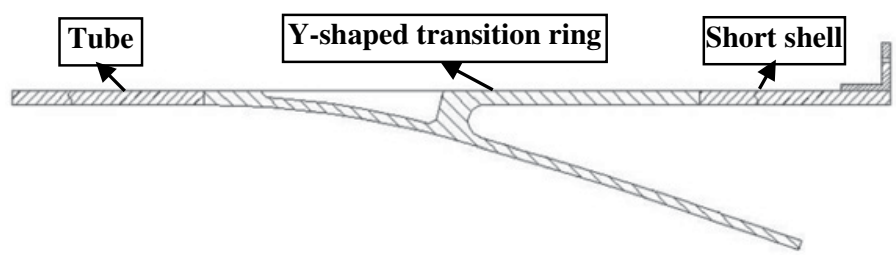

Figure 1. Cross-section of Y-shaped transition ring.

2219-T852

2219-T87

Backing plate

Figure 2. Illustration for welding sample set-up.

3. special-shaped ring integral forging forming + precision bulging, which is developed at the beginning of the 21st century to solve the problems associated with integral forging forming way. It can realized the forming of transition ring with irregular section and accurate control of microstructure and properties.

During the development of $5 \mathrm{~m}$ diameter integral forged Y-shaped transition ring, previous study revealed that microstructure and performance inhomogeneity induced by the increase of structure size. Coarse microstructure is prone to be formed in base material of transition ring and it would affect its mechanical properties and forming performance. Obvious non-uniformity is also found in the welded joint of transition ring, which results in the low elongation with large discreteness, and thus it tends to be the danger zone and restrict the reliability of the tank. In this paper, the integral forging process of 2219 aluminium alloy Y-shaped transition ring for large launch vehicle tank is studied. Microstructure, mechanical properties and welding performance of transition ring are analyzed. Improvement measures for integral forming process of large diameter transition ring are proposed.

\section{Experimental materials and procedure}

2219 aluminium alloy Y-shaped transition ring was produced by reaming and rolling process. The ingot with dimension of $\Phi 940 \mathrm{~mm} \times 2500 \mathrm{~mm}$ was treated by a series of process to obtain the 2219-T852 aluminium alloy transition ring with dimension of $\Phi 5016 \mathrm{~mm}$ (external diameter) $\times \Phi 4748 \mathrm{~mm}$ (inner diameter $) \times 445 \mathrm{~mm}$. It was then processed by machining after qualified by non-destructive testing. Mechanical properties testing samples were taken from the inner part with relatively low properties of transition ring before machining. Y-shaped transition ring and tube was assembled by welding finally.

The welding performance of transition ring with tube was evaluated on test pieces with dimension of $300 \mathrm{~mm} \times 200 \mathrm{~mm} \times 6 \mathrm{~mm}$. 2219-T852 and 2219-T87 aluminium alloy was chosen for transition ring and cube, respectively. The illustration for welding test piece set-up is shown in Fig. 2. Variable polarity pulsed gas tungsten arc welding (VPP-GTAW) process was used. back welding and cosmetic welding were performed under helium and argon gas shielding at flow rate of $11 \mathrm{~L} / \mathrm{min}$, respectively. The detailed welding parameters are listed in Table 1 . The welded joint was evaluated by non-destructive testing after welding.

Microstructural evolution was investigated by optical microscopy (OM, Olympus-PMG3). All OM specimens were prepared by standard metallographic procedures and etched with Keller's reagent $\left(4 \mathrm{ml} \mathrm{HF}+6 \mathrm{ml} \mathrm{HCl}+10 \mathrm{ml} \mathrm{HNO}_{3}+190 \mathrm{ml} \mathrm{H}_{2} \mathrm{O}\right)$. Mechanical properties of base material and weld 
Table 1. Welding parameters for VPP-GTAW process.

\begin{tabular}{|c|c|c|c|c|c|c|c|}
\hline Parameters & $\begin{array}{c}\text { Electric } \\
\text { current } \\
(\mathbf{A})\end{array}$ & $\begin{array}{c}\text { Power } \\
\text { frequency } \\
(\mathbf{H z})\end{array}$ & $\begin{array}{c}\text { AC } \\
\text { balance } \\
(\boldsymbol{\%})\end{array}$ & $\begin{array}{c}\text { Pulse } \\
\text { frequency } \\
(\mathbf{H z})\end{array}$ & $\begin{array}{c}\text { Pulse } \\
\text { duration } \\
(\boldsymbol{\%})\end{array}$ & $\begin{array}{c}\text { Background } \\
\text { current } \\
(\boldsymbol{\%})\end{array}$ & $\begin{array}{c}\text { Welding } \\
\text { speed } \\
(\mathbf{m} / \mathbf{h})\end{array}$ \\
\hline Backing & 210 & - & - & - & - & - & 16 \\
\hline Cosmetic & $\begin{array}{c}\mathrm{EN}: 235 \pm 10 \\
\text { EP:275 } \pm 10\end{array}$ & 55 & 60 & 0.8 & 60 & 50 & 8 \\
\hline
\end{tabular}

Table 2. Tensile properties of test samples taken from different directions of transition ring.

\begin{tabular}{|c|c|c|c|c|c|c|c|}
\hline \multirow{2}{*}{ No. } & \multicolumn{3}{|c|}{ Longitudinal direction } & \multirow{2}{*}{ No. } & \multicolumn{3}{|c|}{ Transverse direction } \\
\cline { 2 - 3 } \cline { 6 - 8 } & $\begin{array}{c}\sigma_{0.2} \\
(\mathrm{Mpa})\end{array}$ & $\begin{array}{c}\sigma_{\mathrm{b}} \\
(\mathrm{Mpa})\end{array}$ & $\begin{array}{c}\delta_{5} \\
(\%)\end{array}$ & & $\begin{array}{c}\sigma_{0.2} \\
(\mathrm{Mpa})\end{array}$ & $\begin{array}{c}\sigma_{\mathrm{b}} \\
(\mathrm{Mpa})\end{array}$ & $\begin{array}{c}\delta_{5} \\
(\%)\end{array}$ \\
\hline $1-1$ & 372 & 464 & 12 & $1-2$ & 369 & 458 & 5 \\
\hline $2-1$ & 371 & 463 & 11 & $2-2$ & 372 & 462 & 6.5 \\
\hline $3-1$ & 364 & 458 & 12.5 & $3-2$ & 367 & 444 & 4.5 \\
\hline $4-1$ & 362 & 456 & 12.5 & $4-2$ & 366 & 432 & 4.5 \\
\hline $5-1$ & 364 & 457 & 10 & $5-2$ & 368 & 452 & 6 \\
\hline $6-1$ & 364 & 458 & 11.5 & $6-2$ & 368 & 449 & 5.5 \\
\hline $7-1$ & 352 & 458 & 11 & $7-2$ & 351 & 444 & 7 \\
\hline $8-1$ & 342 & 452 & 13 & $8-2$ & 351 & 444 & 6 \\
\hline Average & 361 & 458 & 11.6 & Average & 364 & 448 & 5.6 \\
\hline
\end{tabular}

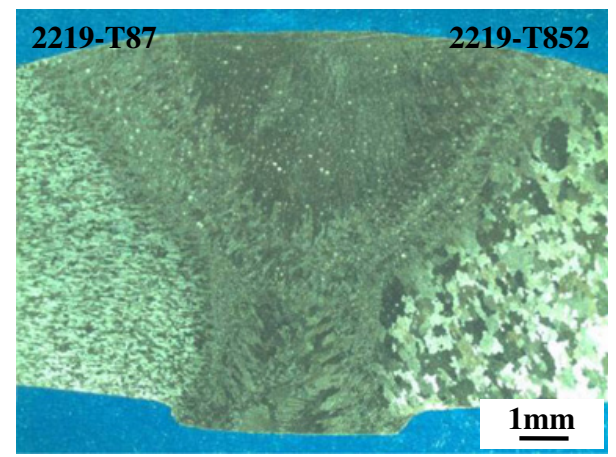

Figure 3. A typical cross-section of 2219-T852/2219-T87 aluminium alloy welded joint.

joints are evaluated by tensile test. Tensile tests were carried out on an Instron-1186 mechanical tester at room temperature using a crosshead speed of $0.5 \mathrm{~mm} / \mathrm{min}$.

\section{Results and discussion}

Tensile properties of test samples for the transition ring are listed in Table 2. It is indicated that mechanical properties of transition ring base material show some differences with different direction, especially for the elongation. The tensile and yield strength of base material are approximately the same for both directions, but elongation of samples for longitudinal direction is almost twice of that for transverse direction.

A typical cross-section of 2219-T852/2219-T87 aluminium alloy welded joint is shown in Fig. 3. It is observed that the grain size is coarser in the HAZ of 2219-T852 aluminium alloy side for the transition ring, which could be attributed to the coarse microstructure in the base material of the transition ring. In addition, grain size inhomogeneity was confirmed in the base material of the transition ring with very coarse grains. 


\section{MATEC Web of Conferences}

Table 3. Mechanical properties and fracture locations of 2219-T852/2219-T87 aluminium alloy welded joints.

\begin{tabular}{|l|l|l|l|l|}
\hline No. & $\begin{array}{l}\sigma_{\mathbf{0 . 2}} \\
\mathbf{M P a}\end{array}$ & $\begin{array}{l}\sigma_{\mathbf{b}} \\
\mathbf{M P a})\end{array}$ & $\begin{array}{l}\boldsymbol{\delta}_{\mathbf{5}} \\
(\mathbf{\%})\end{array}$ & Fracture locations \\
\hline 1 & 155 & 291 & 6.52 & Weld zone with crack initiation at fusion line of 2219-T852 side \\
\hline 2 & 156 & 263 & 5.40 & Weld zone with crack initiation at fusion line of 2219-T852 side \\
\hline 3 & 173 & 289 & 5.28 & Fusion line at 2219-T852 side \\
\hline 4 & 158 & 288 & 6.44 & Weld zone with crack initiation at fusion line of 2219-T852 side \\
\hline 5 & 155 & 268 & 5.24 & Weld zone with crack initiation at fusion line of 2219-T852 side \\
\hline 6 & 157 & 300 & 8.20 & Weld zone with crack initiation at fusion line of 2219-T852 side \\
\hline 7 & 153 & 304 & 8.48 & Fusion line at 2219-T87 side \\
\hline 8 & 158 & 308 & 6.68 & Fusion line at 2219-T87 side \\
\hline Average & 158 & 288 & 6.5 & - \\
\hline
\end{tabular}

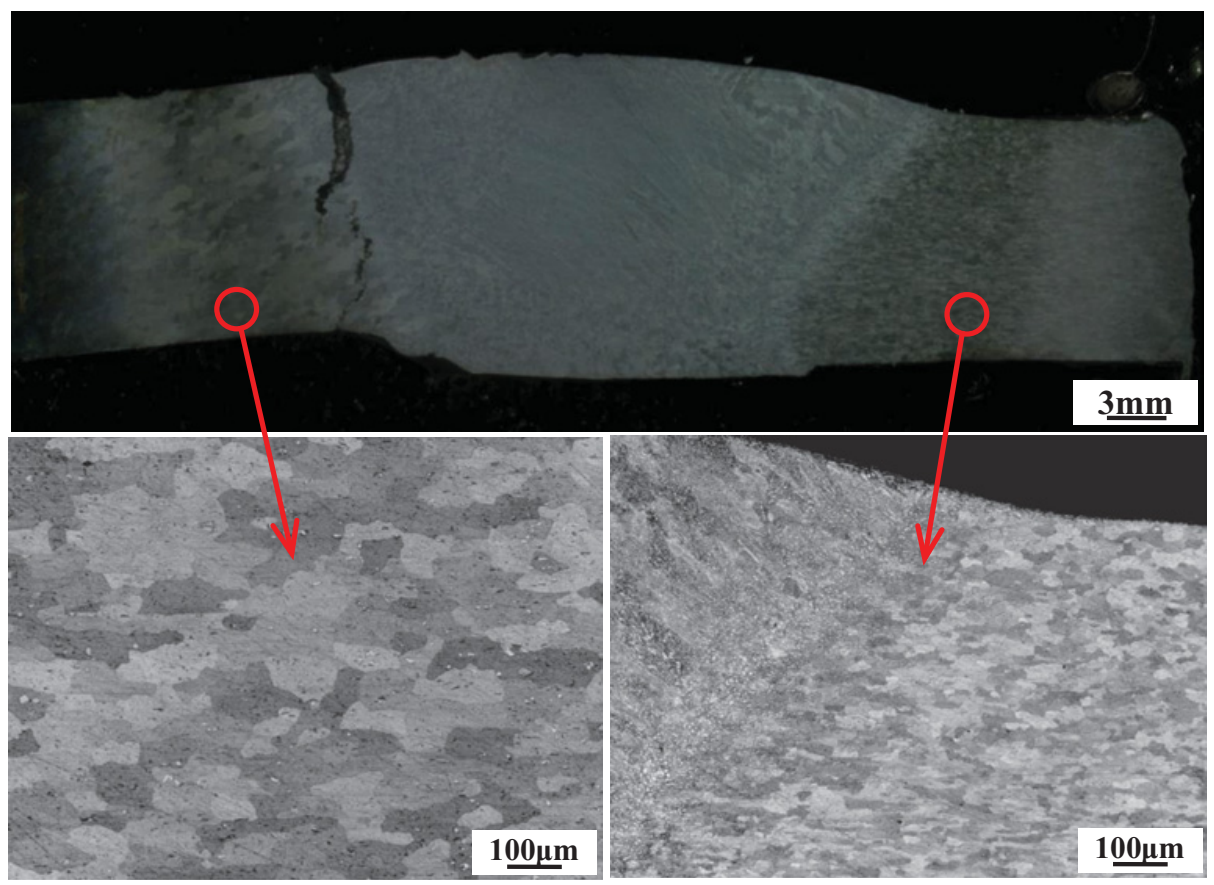

Figure 4. Microstructure of fracture location in 2219-T852/2219-T87 aluminium alloy welded joint.

Mechanical properties and fracture locations of 2219-T852/2219-T87 aluminium alloy welded joints are summarized in Table 3. It is shown that almost all of the welded joints fractured near the fusion line at 2219-T852 aluminium alloy side for the transition ring.

A typical fractured welded joint is shown in Fig. 4, which indicated that the fracture occurred in the 2219-T852 aluminium alloy side consisted of coarse microstructure.

It can be confirmed that degraded microstructure was formed in the region within $1 \mathrm{~mm}$ from fusion line at 2219-T852 aluminium alloy side due to coarse grains in the transition ring. The degraded microstructure was formed perpendicular to the surface of base material, as shown in Fig. 5. Degraded microstructure is the mixture of crisp-hard $\mathrm{Cu}$-rich eutectic phase and soft $\mathrm{Cu}$-leak $\alpha$ phase formed at the grain boundary by liquidation during the welding process, as shown in Fig. 6 . The $\alpha$ phase would be yielded and eutectic phase will be brittle fractured under very low level of stress, which decreases the strength and toughness of welded joints. 


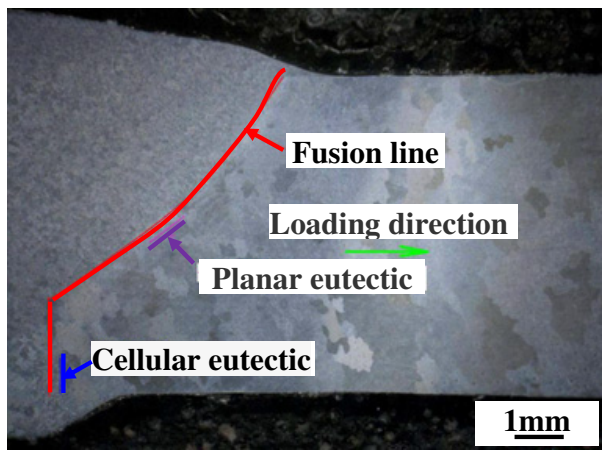

Figure 5. The microstructure and loading direction of 2219-T852/2219-T87 aluminium alloy welded joint.

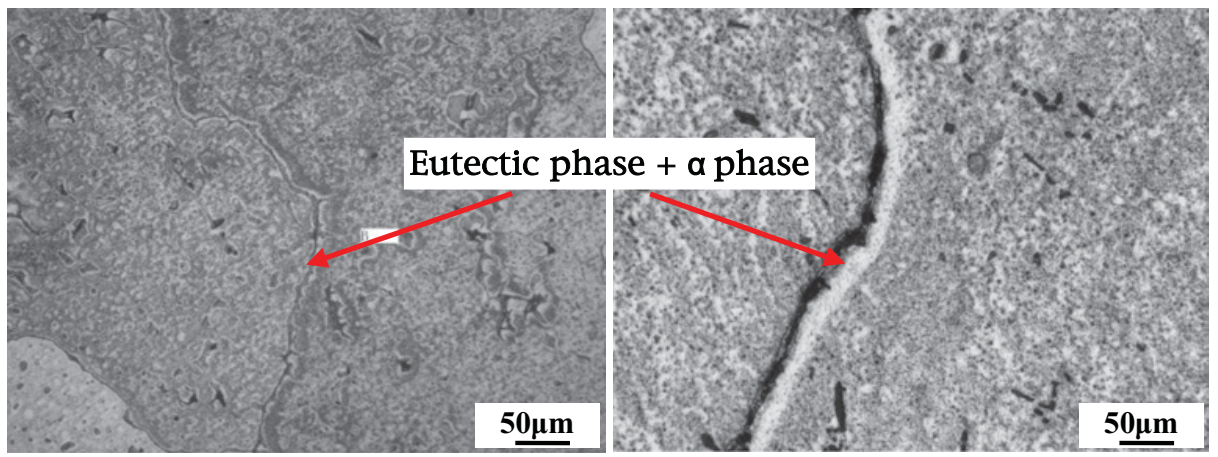

Figure 6. Degraded microstructure of 2219-T852/2219-T87 aluminium alloy welded joint.

\section{Conclusion}

With the development of heavy-lift launch vehicle technology, the development of ultra-large diameter Y-shaped transition ring will be the major key technology to be solved for ultra-large tank in the future. As for manufacture of transition ring for larger launch vehicle tank, the integral forming will be more difficult. As it is currently known, there are two alternatives:

1). the integral forging forming, which can ensure the accurate forming and assembly to the largest degree, and is very benefit for the reliability of large structure.

2). subsection welding by advanced welding technologies like friction stir welding into the integral circular structure at first and then make the transition ring connected by integral machining, which requires relatively low for process and equipment.

No matter what kind of way for forming, the uniformity of performance and the stability of welded joint of larger transition ring are still difficulties and further research work is needed.

\section{References}

[1] Editorial Committee for A Complete Collection of Aerospace Vehicle in the World. A Complete Collection of Aerospace Vehicle in the World (China Aerospace Press, Beijing, 2007) 
[2] J.C. Pilet, D. Diecidue-Conners, M. Worden, Michelle Guillot, W. Kenneth. NASA Report Document with ID: 20120001570 (2012)

[3] W. Robert, R. E. Carter. NASA Report Document with ID: 20120014602 (2012)

[4] R. E. Jones. Assemblies for the NASA Ares I Rocket. NASA Report Document with ID: 20120014528 (2012)

[5] M. Windisch, D.Z. Sun, D. Memhard. Eng. Fract. Mech. 76: 59 (2009)

[6] http://www.spacesafetymagazine.com/aerospace-engineering/rocketry/slsexternal-tank-reverts-hard-alloy/

[7] http://www.nasa.gov/centers/marshall/news/news/releases/2013/13-080.html

[8] B. Hendrickx, B. Vis. Energia-Buran: The Soviet Space Shuttle (Praxis Publishing Ltd, Chichester, 2007)

[9] S.G. Li. Annual Meeting of Professional Committee of Spacecraft Manufacturing Engineering, Chinese Society of Astronautics (2010)

[10] Y.P. Zhu, X.L. Liu. Aerospace Manufacturing Technology 6: 42 (2011) 